\title{
Medical problems in pregnancy
}

\author{
Authors: Bhaskar Narayan ${ }^{\mathrm{A}}$ and Catherine Nelson-Piercy ${ }^{\mathrm{B}}$
}

The prevalence of medical problems in pregnancy is increasing because of a complex interplay between demographic and lifestyle factors, and developments in modern medicine. Maternal mortality and morbidity resulting from treatable medical conditions, such as venous thromboembolism, epilepsy and autoimmune disease, have not decreased in recent years. This is despite a marked decrease in overall maternal mortality. It is vital that all physicians acquire a basic knowledge and understanding of medical problems in pregnancy. This includes prepregnancy measures such as counselling and optimisation of medical therapy, as well as multidisciplinary management throughout pregnancy and the postpartum period. Prompt recognition and treatment of acute and chronic illness is of clear benefit, and most drugs and many radiological investigations may be used in pregnancy.

KEYWORDS: Biologics, maternal mortality, obstetric medicine, pregnancy

\section{Introduction}

The prevalence of medical problems in pregnancy is increasing because of a complex interplay between demographic and lifestyle factors, and developments in modern medicine. Women are delaying childbearing until later in life. Older women are more likely to have acquired medical disorders, such as hypertension and obesity; they are also at higher risk of gestational diabetes and venous thromboembolism. Medical and surgical advances have enabled women to become pregnant despite having chronic conditions that would previously have precluded pregnancy. However, this is not without risk - the MBRRACE (Confidential Enquiries into Maternal Deaths and Morbidity) report ${ }^{1}$ revealed that two-thirds of maternal deaths in 2011-13 were in women with known medical comorbidities and $30 \%$ of mothers that died were obese (Fig 1). Additionally, for every maternal death, there are many others that suffer severe morbidity during or soon after pregnancy. Data from the

Authors: ${ }^{\text {A }}$ clinical fellow in obstetric medicine and specialty registrar in acute medicine and general internal medicine, Women's Health Academic Centre, Guy's and St Thomas' Foundation Trust, London, UK; ${ }^{B}$ consultant obstetric physician and professor of obstetric medicine, Women's Health Academic Centre, Guy's and St Thomas' Foundation Trust, London, UK
Intensive Care National Audit and Research Centre (ICNARC) show that the admission rate to critical care during the period 2009-12 was 290 per 100,000 maternities, compared to a maternal mortality rate of less than 11 per 100,000 over the same period. ${ }^{1}$

Maternal deaths can be classified as direct ('obstetric') or indirect ('medical') (Box 1). As Fig 2 illustrates, there has been a worrying increase in the rate of indirect deaths over the last 25 years and while this has plateaued, direct death rates continue to fall. Medical conditions now dominate the list of leading causes of maternal death (Fig 3). Therefore, it is vital that all physicians acquire a basic knowledge and understanding of medical problems in pregnancy.

The approach to managing medical problems in pregnancy is summarised in Fig 4. The process should begin before conception, and all physicians should consider delivering prepregnancy counselling as part of their routine inpatient or outpatient consultations with women of reproductive age. The MBRRACE reports call for action to 'ensure that physicians are appropriately trained in... the care of pregnant women, and that services are designed for women with medical conditions... including pre-pregnancy, during pregnancy and delivery, and postpartum'. This is clearly a very broad subject, and this article aims to provide a snapshot. We discuss a few of the important causes of maternal mortality, as well as some common medical causes of morbidity that present particular diagnostic and therapeutic challenges in pregnancy.

\section{Box 1. Definitions of maternal deaths (World Health} Organization 2010).

Maternal death: Death of a woman while pregnant or within 42 days of the end of the pregnancy (the puerperium) from any cause related to or aggravated by the pregnancy or its management, but not from accidental or incidental causes.

Direct: Deaths resulting from obstetric complications of the pregnant state (pregnancy, labour and puerperium), from interventions, omissions, incorrect treatment or from a chain of events resulting from any of the above. Examples include genital tract sepsis, hypertensive disorders of pregnancy and thrombosis.

Indirect: Deaths resulting from previous existing disease, or disease that developed during pregnancy and which was not the result of direct obstetric causes, but which was aggravated by the physiological effects of pregnancy. Examples include cardiovascular disease, neurological disorders, non-genital infections and psychiatric causes. 
Fig 1. Selected characteristics of the mothers who died in 2011-13. Adapted from the 2015 MBRRACE report. ${ }^{1}$
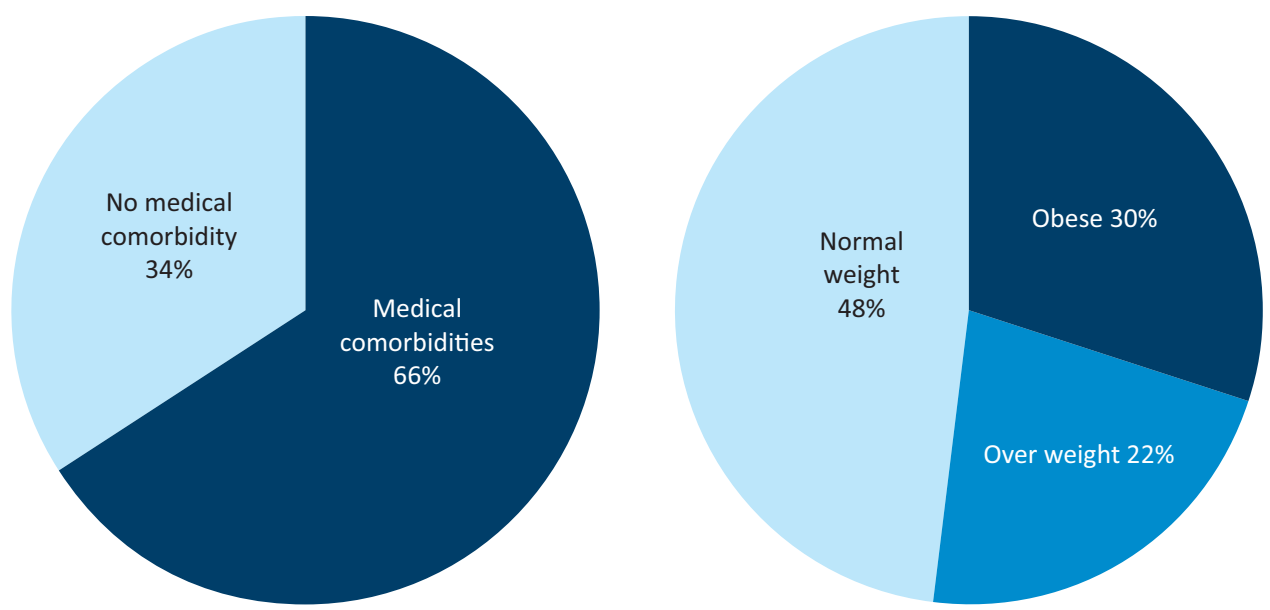

\section{Venous thromboembolism}

Venous thromboembolism (VTE) is the leading direct cause of maternal death in the UK and the trend of falling mortality rates from 2002 to 2008 has reversed in recent years.

The relative risk of VTE in pregnancy is increased four- to sixfold compared with the general population. This risk increases with gestational age, reaching a maximum just after delivery. Caesarean section further increases this risk, particularly if this is performed as an emergency. ${ }^{1}$ Certain women are, because of medical or obstetric factors, at particularly high risk and therefore require antenatal and/or postpartum thromboprophylaxis with low molecular weight heparin (LMWH). LMWH is safe and effective in pregnancy and recent guidelines from the Royal College of Obstetricians and Gynaecologists (RCOG) provide a detailed approach to risk stratification. $^{2}$

As well as preventative measures, it is also important to promptly recognise and treat acute VTE in pregnancy. Venous stasis is more marked in the left leg because of compression of the left iliac vein by the right iliac artery and ovarian artery. $85 \%$ of deep vein thrombosis in pregnancy are on the left side and $70 \%$ are ilio-femoral (which are more prone to lead to pulmonary embolism). ${ }^{3}$

A thorough history and examination is important when assessing for acute pulmonary embolism (PE) because the differential diagnosis includes cardiac disease and aortic dissection. A chest X-ray (CXR) should always be performed first (negligible radiation risk) as pneumonia and pneumothorax can present similarly. If $\mathrm{PE}$ is still suspected, then a nuclear medicine perfusion (VQ) scan or computerised tomography pulmonary angiogram (CTPA) should be performed. Investigation should not be withheld simply because the patient is pregnant. The maternal and perinatal risks of missing a diagnosis of VTE are thought to be greater than the risks associated with the investigations; the risks to the fetus are small with either imaging modality. Compared with CTPA, VQ may carry a very slightly increased risk of childhood cancer $(1 / 280,000$ versus less than $1 / 1,000,000) .{ }^{4}$ However, CTPA is associated with a
Fig 2. Direct and indirect maternal mortality rates per 100,000 maternities in the UK, 1985-2013 (preliminary data 2011-13). Deaths from indirect causes have risen and plateaued in recent years. In contrast, deaths from direct causes are falling. Adapted from the 2015 MBRRACE report.

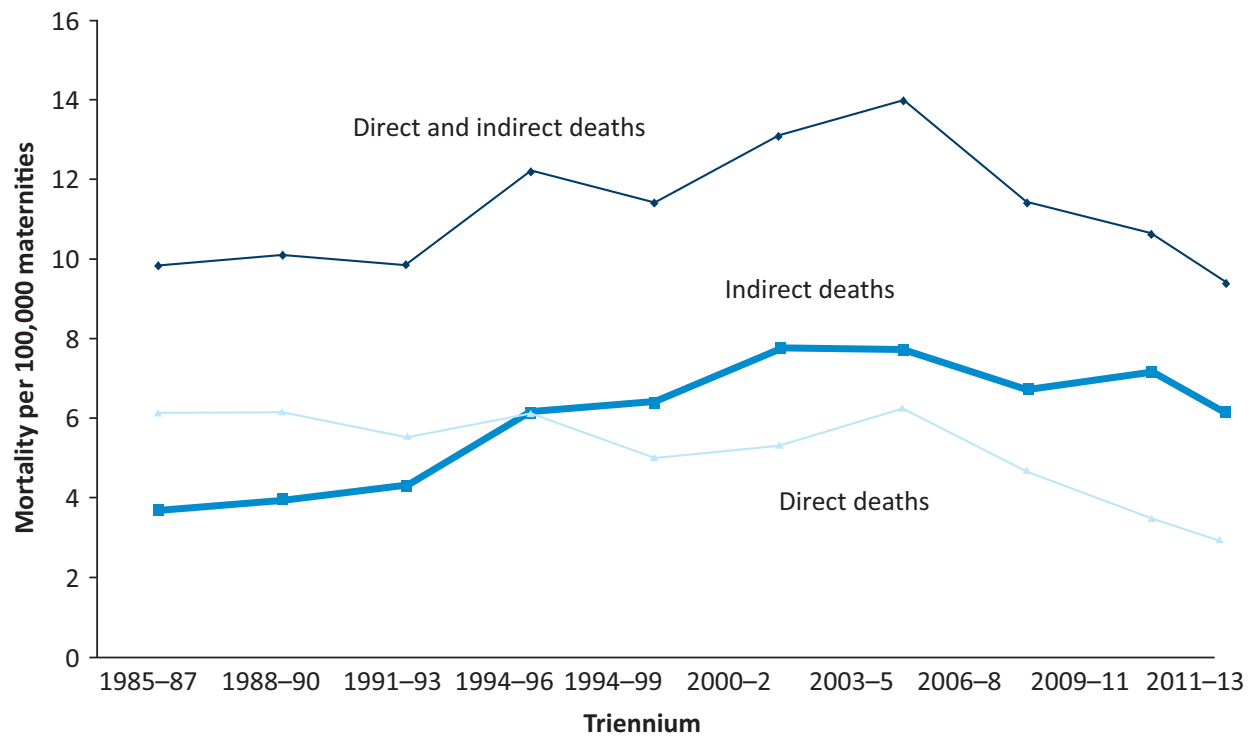




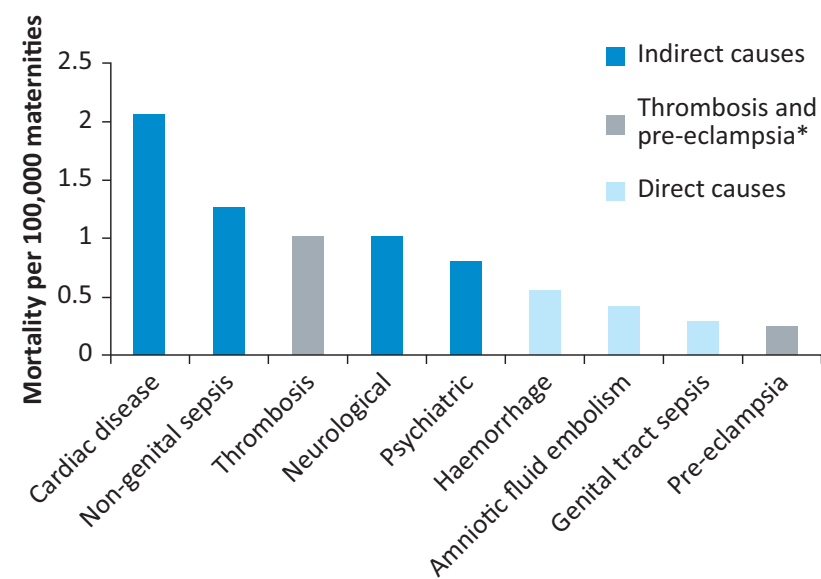

Fig 3. Direct and indirect causes of maternal mortality per 100,000 maternities in the UK, 2011-13. Adapted from the 2015 MBRRACE report. ${ }^{1}$ "Although thrombosis and pre-eclampsia are classified as 'direct', they are often associated with medical risk factors and/or comorbidities.

significantly higher risk of maternal breast cancer (lifetime risk increased by up to $13.6 \%$; background risk of $1 / 200){ }^{5}$

Pre-test probability assessment (eg Wells Score) is not validated in pregnancy and $\mathrm{D}$-dimer testing is not recommended outside the research setting.

Treatment dose LMWH should be commenced immediately and continued if the diagnosis is confirmed. The doses required are higher than in non-pregnant patients and are listed in the RCOG guidelines. ${ }^{6}$ Warfarin should be avoided in pregnancy, as should direct oral anticoagulants such as rivaroxaban. However, pregnancy is not a contraindication to thrombolysis, which can be considered in massive lifethreatening PE.

\section{Epilepsy}

Epilepsy affects $1 \%$ of the UK population and $23 \%$ of people with the condition are women of childbearing age. ${ }^{7}$ There are two main aspects to the management of epilepsy in pregnancy. Firstly, there is the impact of epilepsy on pregnancy outcomes (and vice-versa); secondly, there are the implications of fetal anti-epileptic drug (AED) exposure.

It is essential that epilepsy is recognised as a high-risk condition. Maternal mortality is tenfold higher in patients with epilepsy than in those without the disorder ${ }^{8}$ and more women

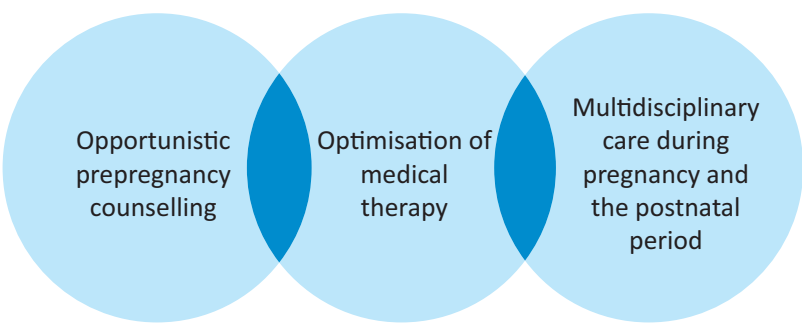

Fig 4. The three aspects of managing women with medical problems in pregnancy. now die from epilepsy in pregnancy than from pre-eclampsia and eclampsia. Epilepsy is also associated with an increased risk of obstetric haemorrhage, hypertensive disorders of pregnancy and fetal growth restriction. ${ }^{9}$

The 2014 MBRRACE report ${ }^{6}$ highlighted that the majority of women who died from epilepsy-related causes had poorly controlled epilepsy prepregnancy and very few had received prepregnancy counselling. This emphasises the need to initiate the process before conception. Because a significant proportion of pregnancies are unplanned, it should be assumed that all women of childbearing age with a diagnosis of epilepsy may become pregnant at some stage in their life. This should prompt opportunistic prepregnancy counselling at the time of diagnosis or initiation of drug treatment and at subsequent contacts with healthcare professionals.

Most women with epilepsy will not experience a change in seizure frequency during pregnancy and women who have been seizure-free for several years are unlikely to have seizures in pregnancy. ${ }^{3}$ Those with poorly controlled epilepsy are at the highest risk of deterioration, injury and death due to SUDEP (sudden unexpected death in epilepsy). However, even in those with well-controlled disease, deterioration may occur following discontinuation of anti-epileptic medications, or if there is a significant fall in drug levels. This may occur because of poor compliance, nausea and vomiting, and altered pharmacokinetics. Pregnancy results in an increased volume of distribution and increased hepatic and renal clearance of many drugs. Lamotrigine, in particular, may require significant dose increases in pregnancy. The results of a large multicentre study (EMPiRE) may clarify the role of therapeutic drug monitoring. All AEDs are teratogenic (Table 1) but the risks of uncontrolled epilepsy almost always outweigh the risks to the fetus posed by these medications. Sodium valproate carries the highest risk of malformations and is also associated with impaired childhood psychomotor development. It is usually switched to an alternative drug pre-conception. Women of childbearing age should only be commenced on sodium valproate if there is no other suitable treatment option. Other anti-epileptics are usually continued through pregnancy although careful counselling is required to ensure that the patient understands the benefits and risks.

\section{Headache}

Headache is a common complaint in the general population; patients presenting to the emergency department with headache are disproportionately female (71\%) and between 18 and 49 years of age $(71 \%) .^{10}$ Therefore, it is unsurprising that it is a relatively frequent presentation in pregnant women. The vast majority of headaches are self-limiting and benign in aetiology but there are some potentially serious causes to be considered. Certain causes of headache warrant a particularly high index of suspicion in pregnant patients.

It is vital to take a thorough history from any patient presenting with headache. Physical examination is often unremarkable and a good history can, in most cases, provide the information required to formulate a working diagnosis.

Primary headaches, such as migraine and tension headache, account for most headaches in pregnancy. The majority of women with pre-existing migraine notice a reduction in frequency and severity of attacks, possibly due to a reduction 
Table 1. Anti-epileptic drugs (AEDs) in pregnancy

Drug Pharmacokinetic considerations in
pregnancy

Sodium valproate

Phenytoin

Lamotrigine

Carbamazepine,

levetiracetam

Benzodiazepines (diazepam, lorazepam, clobazam)
Highly protein-bound; dose increases rarely required

Dose may need to be increased two- to threefold

Drug levels and dose adjustment recommended only if there is a change in seizure frequency

\section{Teratogenic effects}

High risk (4.7-13.8\%) of major fetal malformations (neural tube defects, congenital heart defects)

High rate (30-40\%) of impaired psychomotor development and reduced IQ in the child; risk of childhood autism increased fivefold

The risks are dose-dependent and increase when valproate is combined with other AEDs

Increased risk of congenital heart defects

Alternative drugs should be used where possible

Associated with fetal malformations, but risk appears relatively low. If epilepsy is well controlled on these drugs then they should be continued

Not teratogenic in monotherapy, but in most circumstances used as add-on therapy with another AED in reproductive hormonal fluctuation. However, around 10\% experience a worsening of symptoms and migraine may present de novo in pregnancy. ${ }^{11}$

If clinical assessment supports a diagnosis of migraine or tension headache, then no further investigations are required. Lifestyle measures and simple analgesia are the first-line treatments. Patients should be advised to get adequate sleep and to avoid dietary triggers and dehydration. Paracetamol is acceptable for use in pregnancy and ibuprofen can be prescribed for short-term use in the first and second trimesters. Opiates are not harmful to the fetus but should be used sparingly because of their side-effect profile and tendency to cause rebound headaches. Metoclopramide, cyclizine or prochlorperazine can be added to treat nausea and vomiting.

There are increasing safety data on triptans to treat migraine in pregnancy. ${ }^{12,13}$ Sumatriptan exposure has not been associated with fetal malformations or adverse outcomes and, if other treatments have failed, it may be used to treat acute migraine attacks.

If attacks are frequent, then prophylactic medication should be considered. Low-dose aspirin is the first-line agent. $\beta$-blockers (eg propranolol) can be added and, if this is not effective, then a tricyclic antidepressant (eg amitryptilline) can be used as a third-line option.

It is important to consider secondary causes in the differential diagnosis of headache, which may require urgent investigation. Pre-eclampsia may present with headache and meningitis presents as headache with fever and neck stiffness. Pregnancy significantly increases the risk of subarachnoid haemorrhage and cerebral venous thrombosis. The risk is highest in the puerperium (first 6 weeks post-delivery).

Urgent imaging should be performed in patients who present with severe thunderclap headache, features of raised intracranial pressure, neurological deficits or seizures. CT brain is safe in pregnancy, readily available and indeed superior to MRI in detecting acute haemorrhage. The radiation dose is small and the fetal exposure is negligible. However, magnetic resonance venogram is the preferred imaging modality to detect cerebral venous thrombosis.

Reversible cerebral vasoconstriction syndrome is a recently recognised disorder of cerebrovascular tone, which can cause severe sudden onset headache and atypical subarachnoid haemorrhage in the postpartum period. ${ }^{14}$ It may be associated with hypertension. Diagnosis is on MRI angiography (with a characteristic appearance of vascular beading) and treatment is with nimodipine. It usually resolves within 3 months.

\section{Inflammatory and autoimmune conditions}

Systemic inflammatory diseases, such as systemic lupus erythromatosis, rheumatoid arthritis and inflammatory bowel disease, are relatively common in women of childbearing age. The approach shown in Fig 4 is particularly relevant for women with these conditions.

Good disease control is of obvious benefit to the mother but it also reduces adverse fetal and neonatal outcomes as well as improving fertility. ${ }^{15}$ A 2013 meta-analysis showed that women who conceive with active inflammatory bowel disease have a higher rate of active disease during pregnancy (46-55\%) than those who conceive while in remission $(23-29 \%) .{ }^{16}$ Similarly, systemic lupus erythromatosis flares during pregnancy are more common in those with active disease at conception. ${ }^{17}$ A major source of uncertainty for patients and healthcare professionals relates to the safe use of medications during pregnancy. Very few drugs are licensed for use in pregnancy, so safety information is often derived from animal studies, case series, registry data and extrapolation from non-pregnant subjects. The US Food and Drug Administration recently discontinued the traditional pregnancy categories A, B, C, D and $\mathrm{X}$ as they were often misinterpreted and misused. There is growing evidence of drug safety in pregnancy and it is vital to avoid cessation of important medications because of theoretical 
or unwarranted concerns. It is also important to note that there is a background incidence of major congenital malformations $(2-3 \%)$, miscarriage $(10-20 \%)$ and stillbirths $(0.5 \%){ }^{18}$ This is irrespective of any drug exposure. This section aims to provide an update on the pharmacological treatment of inflammatory disorders in pregnancy.

\section{Corticosteroids}

Prednisolone and methylprednisolone are extensively metabolised by the placenta and less than $10 \%$ reaches the fetus. Several large studies have not found any significant adverse effect (major malformations, prematurity, low birthweight) attributable to these drugs ${ }^{19}$ although there is an increased maternal risk of gestational hypertension and diabetes. Prednisolone should be used in preference to fluorinated steroids, such as dexamethasone, as the latter cross the placenta to a greater degree.

Corticosteroids play a key role in the treatment of disease flares in patients with rheumatological conditions or inflammatory bowel disease as well as acute asthma. They should be used at any stage of pregnancy if clinically indicated.

There is evidence that physicians under prescribe systemic steroids when managing pregnant patients with asthma, resulting in worse outcomes for these women; ${ }^{20}$ it is important to avoid this error when managing pregnant patients with any condition requiring steroid treatment.

\section{Immunomodulators and immunosuppressants}

Many of the drugs used in autoimmune, rheumatological and inflammatory bowel disease can be used in pregnancy. Table 2 shows a summary for commonly used drugs and counselling about pregnancy should be undertaken when these drugs are initiated. There are good safety data for hydroxychloroquine, sulfalazine, mesalazine and azathioprine, none of which are associated with any increase in congenital malformation rates or pregnancy loss. Although azathioprine was, in one study, ${ }^{21}$ associated with neonatal anaemia, this was a small uncontrolled study and the results were not significant after adjustment for

Table 2. Immunosuppressants in pregnancy

$\begin{array}{lll}\begin{array}{l}\text { Acceptable at } \\ \text { all stages of } \\ \text { pregnancy }\end{array} & \begin{array}{l}\text { Acceptable in early } \\ \text { pregnancy (but } \\ \text { can be continued if } \\ \text { required for disease } \\ \text { control) }\end{array} & \begin{array}{l}\text { Avoid in all stages } \\ \text { of pregnancy }\end{array} \\ \text { Infliximab - stop at } & \text { Methotrexate } \\ \text { Prednisolone } & \begin{array}{l}\text { Adalimumab - stop at } \\ 28 \text { weeks if possible }\end{array} & \text { Leflunomide } \\ \text { Ciclosporin } & \text { Etanercept - stop at } & \text { Cyclophosphamide } \\ \text { Tacrolimus } & 28 \text { weeks if possible } & \\ \text { Hydroxychloroquine } & & \\ \text { Sulfasalazine } & & \\ \text { Mesalazine } & & \\ \text { Azathioprine } & & \end{array}$

gestational age. ${ }^{22}$ A prospective study is currently underway to further evaluate this.

The calcineurin inhibitors ciclosporin and tacrolimus are also safe for use in pregnancy. However, drug levels should be monitored closely as drug metabolism and clearance may vary during and after pregnancy. Maternal blood pressure, blood glucose and renal function should also be checked regularly.

The drugs that should be avoided in pregnancy are methotrexate, mycophenolate mofetil, leflunomide and cyclophosphamide. Adequate contraception is imperative.

These drugs should be stopped prior to conception and changed to an alternative medication. It is important to address this in prepregnancy counselling and to aim for optimal disease control on the new drug prior to conception. In exceptional circumstances, cyclophosphamide may be used in the second and third trimesters for conditions such as breast cancer and rapidly progressive interstitial lung disease. ${ }^{23}$

\section{Biologics}

'Biologic' anti-TNF $\alpha$ drugs, such as infliximab, adalimumab and etanercept, have revolutionised the treatment of systemic inflammatory disorders. All three can be safely used in early pregnancy; they do not cross the placenta until after 16 weeks of gestation. There are no significant associations with a particular pattern of congenital malformations or adverse pregnancy outcomes. Beyond 16 weeks, decisions about continuation are individualised based on the drug clearance rate, degree of placental transfer and the patient's clinical condition.

Infliximab and adalimumab are immunoglobulin $\mathrm{G}$ antibodies and are therefore actively transported across the placenta from 16 weeks, resulting in higher drug levels in the neonate than in the mother. Etanercept appears to have a lower rate of placental transfer.

To minimise neonatal levels at birth, these drugs should be discontinued, if possible, by 20 weeks for infliximab and 28 weeks for adalimumab or etanercept. However, in some patients with active inflammatory disease, it is acceptable and indeed necessary to continue treatment throughout pregnancy. The British Society of Rheumatologists and European League Against Rheumatism have recently issued detailed guidance ${ }^{24,25}$ on this topic.

If infliximab is used throughout pregnancy, it can take up to 7 months for blood levels to become undetectable in the infant. Adalimumab has a slightly shorter duration of action and takes up to 11 weeks to become undetectable. The effect on the neonate is unclear and there are theoretical concerns about neonatal immunosuppression. However, data from the PIANO registry ${ }^{26}$ are reassuring: third trimester anti-TNF $\alpha$ use had no effect on infant growth, development and immune development in the first year of life and a systematic review ${ }^{27}$ found no increased risk of infections up to 1 year of age. There has been a single isolated case of fatal disseminated Bacillus Calmett-Guerin (BCG) in an infliximab-exposed infant who received the vaccine at 3 months of age. It is therefore recommended that infants exposed to antiTNF $\alpha$ drugs should not receive live vaccines in the first 6 months of life. All of these drugs may be detectable in breast milk at very low levels, but they are very poorly absorbed via the oral route so breastfeeding is considered safe.

There are a number of newer anti-TNF $\alpha$ drugs, some of which have been modified to alter the pharmacokinetic profile. 
Table 3. Antihypertensive drugs in pregnancy

\begin{tabular}{lllll} 
1st choice & Alternative first-line & Second-line & Third-line & Contraindicated in pregnancy \\
Labetalol & Nifedipine (MR) & Methyldopa & Doxazosin & ACE inhibitors (safe when breastfeeding*) \\
& Amlodipine & Hydralazine & Angiotensin II receptor antagonists Spironolactone \\
& & Selective $\beta$-blockers & \\
\hline & &
\end{tabular}

Certolizumab pegol is an antigen-binding fragment of a monoclonal anti-TNF $\alpha$ antibody (lacking the Fc region) that has been conjugated with poly-ethylene glycol. It has low rates of placental transfer and early data suggest that it is compatible with all three trimesters of pregnancy.

\section{Hypertensive disorders of pregnancy}

Hypertension is the most common medical problem in pregnancy. In normal pregnancy, blood pressure falls by around $10 \mathrm{mmHg}$ in the first half of pregnancy, and then gradually returns to prepregnancy levels by term. Hypertension in pregnancy increases the risk of preeclampsia, preterm delivery, fetal growth restriction, placental abruption and perinatal death. Women may have pre-existing hypertension or gestational hypertension (developing after 20 weeks of gestation). Pre-eclampsia is a disease specific to pregnancy and can be defined as new/worsening hypertension with significant proteinuria or organ dysfunction (Fig 5). ${ }^{28}$ Pre-eclampsia often occurs de novo, but women with preexisting hypertension may develop superimposed preeclampsia, and gestational hypertension may also evolve into pre-eclampsia. It requires hospital admission for close monitoring and the only cure is delivery, which may be required urgently in severe cases. Pre-eclampsia is associated with a doubling of the lifetime risk of developing hypertension, coronary artery disease, cerebrovascular disease and chronic kidney disease. In women with early-onset pre-eclampsia (before 34 weeks), the risk is increased up to eightfold.

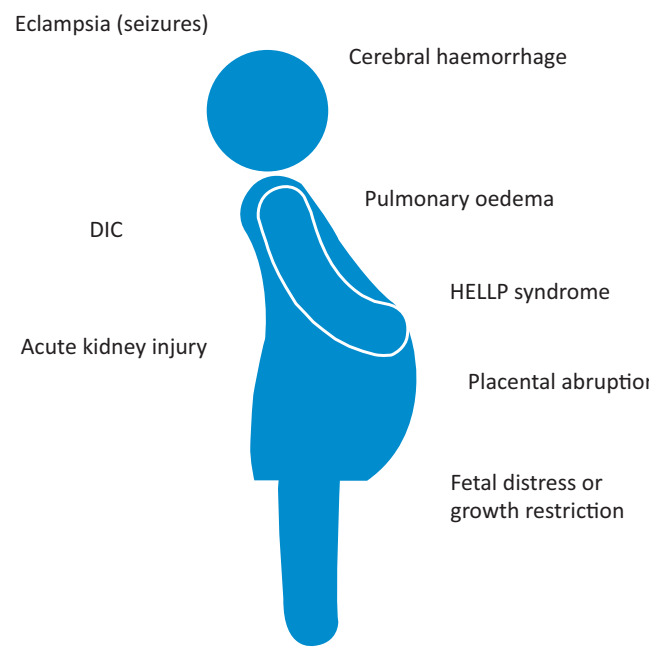

Fig 5. Major complications of pre-eclampsia. DIC = disseminated intravascular coagulation; HELLP = haemolysis elevated liver enzymes low platelets
Therefore, a pregnancy history should be taken when assessing cardiovascular risk in women.

It is common practice to aim for target blood pressure lower than $150 / 100 \mathrm{mmHg}$ in pregnancy and this is consistent with the 2010 National Institute for Health and Care Excellence guidelines. ${ }^{29}$ There were theoretical concerns that lower targets might compromise uteroplacental circulation. However, recent evidence from the CHIPS trial ${ }^{30}$ showed that neonatal outcomes are no different with a target diastolic blood pressure of $81-85 \mathrm{mmHg}$ compared with 100-104 $\mathrm{mmHg}$ and severe maternal hypertension was more frequent in the less tight control (100-104 mmHg) group. Therefore, when treating hypertension in pregnancy, the aim should now be tight control, with a target diastolic blood pressure of $81-85 \mathrm{mmHg}$. If pharmacological treatment is required, labetalol (nonselective $\alpha$ - and $\beta$-blocker) or modified-release nifedipine (calcium channel blocker) are the first-line drugs in pregnancy (Table 3). Methyldopa and amlodipine are second-line options. Importantly, angiotensin-converting enzyme inhibitors, angiotensin II receptor blockers and spironolactone are contraindicated in pregnancy and should be switched (ideally pre-conception) to an alternative agent. However, angiotensinconverting enzyme inhibitors can be reintroduced postpartum and are safe when breastfeeding. ${ }^{31}$

\section{Conclusions}

Maternal mortality and morbidity resulting from treatable medical conditions have not decreased in recent years, despite a marked decrease in overall maternal mortality. Part of the solution is to improve the training of physicians in the management of pregnant patients, including pre-conception counselling. Prompt recognition of acute illness and optimal treatment of chronic conditions is of clear benefit, and most drugs and many radiological investigations can be used in pregnancy.

\section{Conflicts of interests}

The authors have no conflicts of interests to declare.

\section{Note}

This article was originally published in the 2016 Clinical Medicine supplement Horizons in Medicine 28. All articles in this supplement are available at www.clinmed.rcpjournal.org/content//16/Suppl_6

\section{References}

1 Knight M, Tuffnell D, Kenyon S et al. Saving lives, improving mothers' care: surveillance of maternal deaths in the UK 2011-13 and lessons learned to inform maternity care from the UK and Ireland Confidential Enquiries into Maternal Deaths and Morbidity 2009-13. Oxford: University of Oxford, 2015. 
2 Royal College of Obstetricians and Gynaecologists. Reducing the risk of venous thromboembolism during pregnancy and the puerperium. RCOG green-top guideline No 37a. London: RCOG, 2015.

3 Nelson-Piercy C. Handbook of obstetric medicine, 5th edn. Boca Raton, FL: CRC Press, 2015.

4 Cook JV, Kyriou J. Radiation from CT and perfusion scanning in pregnancy. BMJ 2005;331:350.

5 Remy-Jardin M, Remy J. Spiral CT angiography of the pulmonary circulation. Radiology 1999;212:615-36.

6 Royal College of Obstetricians and Gynaecologists. Thromboembolic disease in pregnancy and the puerperium: acute management. RCOG green-top guideline No 37b. London: RCOG, 2015.

7 Kelso A, Wills A on behalf of the MBRRACE-UK neurology chapter writing group. Learning from neurological complications. In: Knight $\mathrm{M}$, Kenyon S, Brocklehurst P et al. Saving lives, improving mothers' care: lessons learned to inform future maternity care from the UK and Ireland Confidential Enquiries into Maternal Deaths and Morbidity 2009-12. Oxford: University of Oxford, 2014:73-79.

8 Edey S, Moran N, Nashef L. SUDEP and epilepsy-related mortality in pregnancy. Epilepsia 2014;55:e72-4.

9 Viale L, Allotey J, Cheong-See F et al. Epilepsy in pregnancy and reproductive outcomes: a systematic review and meta-analysis. Lancet 2015;386:1845-52.

10 Goldstein JN, Camargo CAJr, Pelletier AJ, Edlow JA. Headache in United States emergency departments: demographics, work-up and frequency of pathological diagnoses. Cephalalgia 2006;26:684-90.

11 Schoen JC, Campbell RL, Sadosty AT. Headache in pregnancy: an approach to emergency department evaluation and management. West J Emerg Med 2015;16:291-301.

12 Ephross SA, Sinclair SM. Final results from the 16-year sumatriptan, naratriptan, and treximet pregnancy registry. Headache 2014;54:1158-72.

13 Marchenko A, Etwel F, Olutunfese O et al. Pregnancy outcome following prenatal exposure to triptan medications: a meta-analysis. Headache 2015;55:490-501.

14 Wiles KS, Nortley R, Siddiqui A, Holmes P, Nelson-Piercy C. Reversible cerebral vasoconstriction syndrome: a rare cause of postpartum headache. Pract Neurol 2015;15:141-4.

15 Brouwer J, Hazes JM, Laven JS, Dolhain RJ. Fertility in women with rheumatoid arthritis: influence of disease activity and medication. Ann Rheum Dis 2015;74:1836-41.

16 Abhyankar A, Ham M, Moss A. Meta-analysis: the impact of disease activity at conception on disease activity during pregnancy in patients with inflammatory bowel disease. Aliment Pharmacol Ther 2013;38:4606.

17 Soh M, Nelson-Piercy C. High-risk pregnancy and the rheumatologist. Rheumatology 2015;54:572-87.
18 UK Teratology Information Service. Available online at www.uktis.org/ [Accessed 5 September 2016].

19 Hviid A, Mølgaard-Nielsen D. Corticosteroid use during pregnancy and risk of orofacial clefts. CMAJ 2011;183:796-804.

20 Hasegawa K, Cydulka RK, Sullivan AF et al. Improved management of acute asthma among pregnant women presenting to the ED. Chest 2015;147:406-14.

21 Jharap B, de Boer NK, Stokkers P et al. Intrauterine exposure and pharmacology of conventional thiopurine therapy in pregnant patients with inflammatory bowel disease. Gut 2014;63:451-7.

22 Wiles K, Chandiramani M, Jiwani S, Nelson-Piercy C. A retrospective study of anaemia in neonates exposed to thiopurines in utero. Gut 2016;65:886-7.

23 Nelson-Piercy C, Agarwal S, Lams B. Lesson of the month: selective use of cyclophosphamide in pregnancy for severe autoimmune respiratory disease. Thorax 2016;71:667-8.

24 Flint J, Panchal S, Hurrell A et al. BSR and BHPR guideline on prescribing drugs in pregnancy and breastfeeding-Part I: standard and biologic disease modifying anti-rheumatic drugs and corticosteroids. Rheumatology 2016;55:1693-7.

25 Götestam Skorpen C, Hoeltzenbein M, Tincani A et al. The EULAR points to consider for use of antirheumatic drugs before pregnancy, and during pregnancy and lactation. Ann Rheum Dis 2016;75:795-810.

26 Mahadevan U, Martin C, Sandler R et al. PIANO: A 1000 patient prospective registry of pregnancy outcomes inwomen with IBD exposed to immunomodulators and biologic therapy. Gastroenterology 2012;142(Suppl 1):S-149.

27 Nielsen OH, Jr Loftus EV, Jess T. Safety of TNF-alpha inhibitors during IBD pregnancy: a systematic review. BMC Med 2013;11:174.

28 Tranquilli AL, Dekker G, Magee L et al. The classification, diagnosis and management of the hypertensive disorders of pregnancy: a revised statement from the ISSHP. Pregnancy Hypertens 2014;4:97-104.

29 National Institute for Health and Care Excellence. Hypertension in pregnancy: diagnosis and management. NICE clinical guideline No 107. Manchester: NICE, 2011.

30 Magee L, von Dadelzen P, Rey E et al. Less tight versus tight control of hypertension in pregnancy. N Engl J Med 2015;372:407-17.

31 US National Library of Medicine. LactMed, a TOXNET database. Available online at https://toxnet.nlm.nih.gov/newtoxnet/lactmed.htm [Accessed 5 September 2016].

Address for correspondence: Professor C Nelson-Piercy, Women's Health Academic Centre, Guy's and St Thomas' Foundation Trust, 10th Floor, North Wing, St Thomas' Hospital, Westminster Bridge Road, London SE1 7EH, UK. Email: Catherine.Nelson-Piercy@gstt.nhs.uk 